\title{
REVIEW
}

\section{DNase trials in cystic fibrosis}

\author{
M.E. Hodson, P.L. Shah
}

DNase trials in cystic fibrosis. M.E. Hodson, P.L. Shah. CERS Journals Ltd 1995. ABSTRACT: Cystic fibrosis (CF) is a disease with a high morbidity and mortality from pulmonary disease. Sputum from CF patients contains high levels of deoxyribonucleic acid (DNA), which contribute to its viscoelasticity. Recombinant deoxyribonuclease (rhDNase) has been developed and in vitro studies have showed reduction in the viscoelasticity of $\mathrm{CF}$ sputum. This article reviews the in vivo clinical trials conducted to determine the safety and efficacy of this treatment.

Phase 1 studies showed preliminary safety data and some evidence of clinical benefit. The two Phase 2 short-term studies showed improvement in pulmonary function and important safety data. The Phase 3 study, which included 968 patients, showed improvement in forced expiratory volume in one second (FEV1) of 5.8\% and $5.6 \%$ in patients treated once and twice daily, respectively. The risk of developing an exacerbation of infection was reduced by $28 \%$ with once daily and $37 \%$ with twice daily treatment, compared to placebo. The drug was safe and there was some improvement in quality of life data.

Longer-term open labelled studies, the results of intermittent administration, administration to severely ill patients, and the use of different delivery systems are reviewed.

In conclusion, recombinant deoxyribonuclease is a new treatment which has been shown to benefit patients with cystic fibrosis when used in conjunction with conventional treatment.

Eur Respir J., 1995, 8, 1786-1791.
Dept of Cystic Fibrosis, Royal Brompton National Heart \& Lung Hospital, London, UK.

Correspondence: M.E. Hodson Dept of Cystic Fibrosis Royal Brompton Hospital Sydney Street London SW3 6NP UK

Keywords: Cystic fibrosis deoxyribonuclease sputum

Received: January 311995 Accepted after revision June 211995
Cystic fibrosis $(\mathrm{CF})$ is the commonest inherited disease of the Caucasian population. Respiratory disease is the major cause of mortality and morbidity [1]. In 1938, nearly $70 \%$ of babies died before the first year of life [2]. Latest figures from the US Cystic Fibrosis Foundation show a $50 \%$ survival to 27.6 yrs [3]. The corresponding figures for the UK show a $50 \%$ survival to $25 \mathrm{yrs}$ [4]. The major cause of death in CF is respiratory failure due to the accumulation of viscid infective secretions obstructing and damaging the airways. This causes a relentless decline in pulmonary function inspite of the best treatment currently available.

Deoxyribonuclease (DNase) is released from degenerating polymorphonuclear leucocytes in the sputum of patients with $\mathrm{CF}$, and the concentration of deoxyribonucleic acid (DNA) in the infected sputum can be very high [5-8]. Many years ago, it was shown that bovine pancreatic DNase 1 reduced the viscosity of sputum [9]. A number of clinical studies also reported a reduction in sputum viscosity [10-14]. However, following the reporting of an adverse respiratory reaction, bovine pancreatic DNase fell into disuse [15].

The gene for human DNase 1 has been cloned from a pancreatic complementary DNA (cDNA) library. Recombinant human DNase 1 (rhDNase) has been expressed in Chinese hamster ovary cells and has been shown to be identical in amino acid sequence to the human enzyme found in the blood, in pancreatic secretions and in saliva [6]. Recombinant DNase reduces the viscoelasticity of $\mathrm{CF}$ sputum in vitro and increases mucociliary transport ability as measured by ciliary transport rate on the frog palate [17]. Recombinant DNase (Dornase alfa) administered by aerosol inhalation, using a jet nebulizer driven by air compressor, has been extensively investigated for safety and efficacy in patients with $\mathrm{CF}$, and the results of these trials will now be reviewed.

\section{Phase 1 trials}

Phase 1 studies were conducted to determine the tolerance to initial and repetitive dosing with rhDNase, the rate of elimination of rhDNase from the expectorated sputum samples, and whether significant absorption of rhDNase occurred after single and multiple doses. Fourteen adult subjects with $\mathrm{CF}$ were treated as in-patients, initially with escalating doses, and this was followed by repeated doses of up to $10 \mathrm{mg}$ t.i.d. for 5 days [18]. These patients tolerated short-term administration of rhDNase with no evidence of bronchoconstriction or other airways reaction attributable to the drug. No significant changes in chest radiographs associated with dosing were documented. 
In a second Phase 1 study involving 32 patients with $\mathrm{CF}$, during the first days of the study three CF patients were treated with escalating doses of rhDNase, up to 20 $\mathrm{mg}$ daily. Subsequently, $13 \mathrm{CF}$ patients received doses of either $20 \mathrm{mg}$ daily, $10 \mathrm{mg}$ t.i.d., or $20 \mathrm{mg}$ b.i.d., in order to assess safety and optimize therapy. Blood was obtained for assessment of serum concentrations of DNase and for determination of antibodies to rhDNase. Sputum was obtained from CF patients, postinhalation, to determine the concentration of rhDNase in the sputum. No evidence of adverse respiratory responses, such as bronchospasm or other airways reactions, were attributable to the drug, and some patients experienced a 10-20\% improvement in FEV1 and forced vital capacity (FVC) compared to when they received placebo. No CF patient developed antibodies to rhDNase during short-term administration [19].

\section{Phase 2 trials}

Phase 2 trials were conducted in the US and the UK. These studies employed a randomized, double-blind, placebo-controlled, parallel-group design of patients with mild or moderate disease having a FVC of $>40 \%$ predicted. All patients were clinically stable with no hospitalization or change in therapy within 14 days of enrolment. All patients continued to receive routine therapy for cystic fibrosis, including chest physiotherapy, bronchodilators or steroids. Treatment groups were compared with respect to the mean percentage change in pulmonary function recorded at intervals during their treatment. FEV1, FVC, and quality of life measurements were also recorded.

The US study was a multicentre, double-blind, controlled trial in which 181 clinically stable CF patients, whose FVC was more than $40 \%$ predicted at baseline and who were 8 yrs of age or older, took part [20]. These patients were randomized to receive placebo, $0.6 \mathrm{mg}$ rhDNase, $2.5 \mathrm{mg}$ rhDNase, or $10 \mathrm{mg}$ rhDNase b.i.d. The initial dose was given under observation, and spirometry obtained before and after dosing. Patients were treated as out-patients for 10 days. All patients received a rechallenge of $10 \mathrm{mg}$ rhDNase 18 days after the last dose of trial medication was given. The primary objectives of the study were to determine the effect of administration of rhDNase on FEV1 and FVC, and to determine whether patients develop drug allergy, antibodies to rhDNase after rechallenge, and to study the effects of rhDNase on quality of life of CF patients.

Administration of rhDNase significantly improved pulmonary function. All three doses of rhDNase significantly improved FEV1 (10-14\%) and FVC (10-12\%) from baseline to day 10 (fig. 1). Four days after the end of the treatment period, the improvements in FEV1 and FVC were declining. Quality of life was assessed using a visual analogue scale for dyspnoea, a self-administered questionnaire to quantitate CF-related symptoms and overall well-being. There was an improvement in quality of life and a reduction in dyspnoea in patients taking rhDNase compared with placebo. There was no significant difference between the administration of rhDNase 10 and $2.5 \mathrm{mg}$ b.i.d.

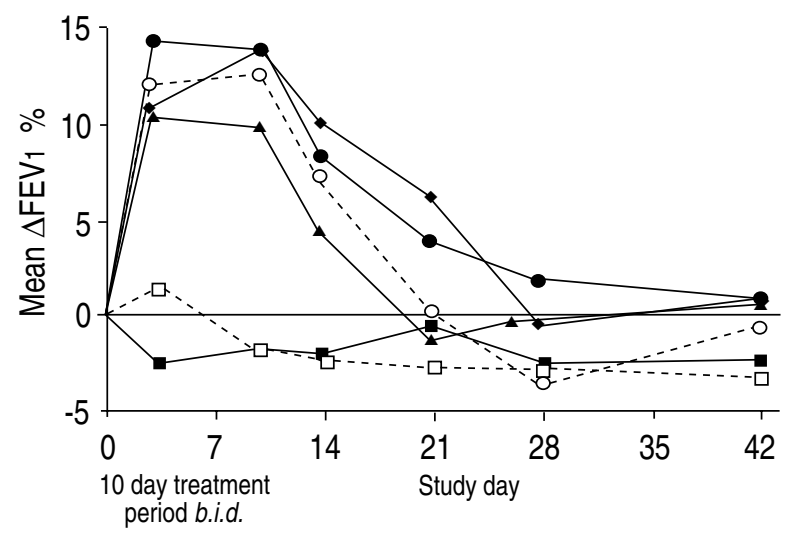

Fig. 1. - Mean percentage change from baseline in forced expiratory volume in one second $\left(\triangle \mathrm{FEV}_{1}\right)$ by treatment group in US and UK phase 2 studies. US study: $\square$ placebo; $\boldsymbol{\Delta}$ :Dornase alfa $0.6 \mathrm{mg}$; $\quad$ : Dornase alfa $2.5 \mathrm{mg} ;$ : Dornase alfa $10.0 \mathrm{mg}$;

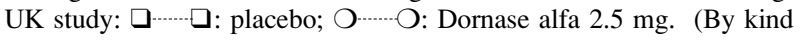
permission of Am Rev Respir Dis [20] and Lancet [21]).

Table 1. - Incidence of adverse events in Phase 2 trial in US

\begin{tabular}{|c|c|c|c|c|}
\hline & Placebo & $\begin{array}{c}0.6 \mathrm{mg} \\
\text { b.i.d. }\end{array}$ & $\begin{array}{c}2.5 \mathrm{mg} \\
\text { b.i.d. }\end{array}$ & $\begin{array}{c}10 \mathrm{mg} \\
\text { b.i.d. }\end{array}$ \\
\hline Adverse event $\mathrm{n}$ & 48 & 45 & 44 & 44 \\
\hline Facial oedema \% & 0 & 0 & 2 & 5 \\
\hline Dyspepsia \% & 0 & 9 & 5 & 7 \\
\hline Voice alteration \% & 0 & 7 & 11 & 16 \\
\hline Pharyngitis \% & 33 & 47 & 64 & 45 \\
\hline
\end{tabular}

Administration of rhDNase did not result in an increased number of serious respiratory events. No patient developed anaphylaxis, signs of drug allergy, or antibodies to rhDNase. Initial challenge and subsequent rechallenge was well-tolerated. There was, however, an increased incidence of voice alteration, pharyngitis, dyspepsia and facial oedema in patients treated with rhDNase compared to placebo (table 1).

The Phase 2 UK study [21] included 71 patients aged 16-55 yrs receiving rhDNase $2.5 \mathrm{mg}$ b.i.d. or placebo for 10 days. Administration of rhDNase significantly improved FEV1 by a mean of $13.3 \%$ over baseline (p<0.001) (fig. 1). The FVC increased by $7.2 \%$, but these results did not achieve statistical significance. The effects subsided within a few days of cessation of therapy. The rhDNase group showed an improvement in their dyspnoea score but this did not reach statistical significance. There was, however, significant improvement in specific variables including "feeling", cough frequency and chest congestion. There was no increase in adverse respiratory events, anaphylaxis, drug allergy or serum antibodies to rhDNase. Rechallenge with rhDNase at day 28 was well-tolerated, and there was no increased frequency of abnormal bronchial reactivity relative to the placebo. Unlike the US Phase 2 study, there was no increase in voice alteration, pharyngitis, dyspepsia or facial oedema in this study.

\section{Intermittent dosing}

An open-labelled study was carried out to assess intermittent therapy with high dose rhDNase in relation to 


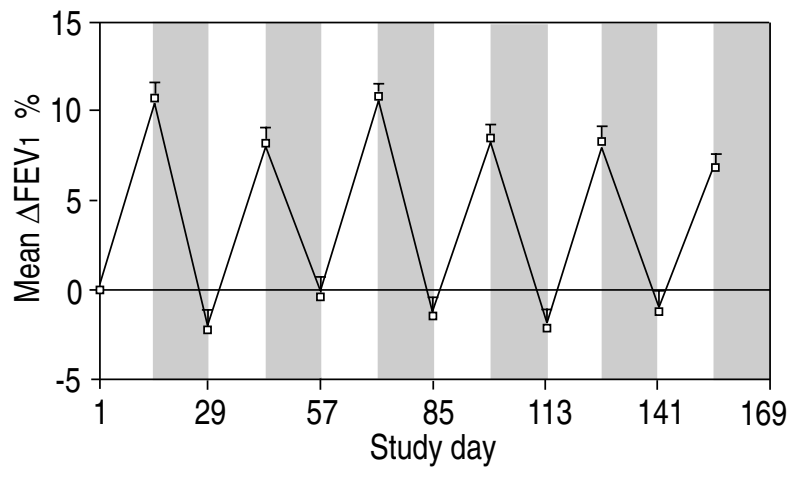

Fig. 2. - Mean percentage change (+SEM) from baseline in forced expiratory volume in one second $(\triangle \mathrm{FEV} 1)$ in Phase $2 \mathrm{~B}$ open-label study of intermittent dosing ( 2 weeks on/2 weeks off) with Dornase alfa $10 \mathrm{mg}$ b.i.d. over 24 weeks [24]. $\square$ : on drug; $\square$ : off drug.

potential allogenic or immunological responses. Patients were given rhDNase $10 \mathrm{mg}$ b.i.d. intermittently (14 days on/14 days off) over 24 consecutive weeks. There were 160 evaluable patients, 12 developed antibodies to rhDNase and were rechallenged under careful clinical observation, but none developed anaphylaxis or a significant decrease in pulmonary function. Each time the patient received therapy, pulmonary function improved over baseline values, but within a short time of discontinuing therapy pulmonary function returned to baseline (fig. 2). This suggested that regular administration of rhDNase is required.

Phase 2 studies indicated that rhDNase improved FEV1 and FVC, dyspnoea, overall well-being and $\mathrm{CF}$ related symptoms. The results were reproducible in different countries. Benefit was obtained within 3 days of starting therapy and was lost quickly when treatment was stopped. The dose of $2.5 \mathrm{mg}$ b.i.d. appeared to be as effective as a higher dose of $10 \mathrm{mg}$ b.i.d.. Intermittent dosing appeared to be safe, but did not maintain improvement in lung function as did regular daily treatment. Other studies were required to study the medium and longer-term effects of administration of rhDNase to patients with mild and moderate $\mathrm{CF}$. Patients with severe pulmonary disease (FVC $<40 \%$ pred) also needed to be studied.

\section{Phase 3 trial}

The Phase 3 study was conducted in 51 centres in the US [22]. Nine hundred and sixty eight CF patients were enrolled in a randomized, double-blind, placebo-controlled trial with parallel-group design of 24 weeks duration. The objectives were to assess the efficacy of rhDNase in reducing the rate of respiratory tract exacerbations requiring parenteral antibiotic therapy, and to evaluate the effect on FEV1, quality of life and safety parameters. Patients were randomized to receive rhDNase $2.5 \mathrm{mg}$ daily, $2.5 \mathrm{mg}$ b.i.d. or placebo. All patients were $\geq 5 \mathrm{yrs}$ of age, of stable pulmonary status, with no exacerbations of pulmonary infection or change in antibiotic regimen within 14 days of enrolment. All had an FVC of $>40 \%$ predicted. All standard CF treatment was continued. A
Table 2. - Protocol-defined respiratory tract infections four or more of these criteria

Change in sputum production (volume, colour or consistency) New or increased haemoptysis

Increased cough

Increased dyspnoea

Malaise, fatigue or lethargy

Fever (more than $38^{\circ} \mathrm{C}$ )

Anorexia or weight loss

Sinus pain or tenderness

Change in sinus discharge

A $10 \%$ decrease in FEV1 for FVC from a previously recorded value

Radiographic changes indicative of pulmonary infection Changes in chest sounds

FEV1: forced expiratory volume in one second; FVC: forced vital capacity.

protocol defined "respiratory tract infection" was said to have occurred when parenteral antibiotics were prescribed for patients having four or more of the characteristics shown in table 2 .

\section{Double-blind period of efficacy result}

The proportion of patients in each treatment group remaining free of protocol-defined respiratory tract infections requiring parenteral antibiotic therapy was plotted over time using Kaplan-Meier curves. The percentage of patients free from protocol-defined respiratory tract infection was: in the rhDNase daily group 78\%; in the rhDNase b.i.d. group $81 \%$; compared with placebotreated patients $73 \%$ (fig. 3). The risk of developing one or more protocol-defined respiratory tract infections requiring parenteral antibiotics over the 24 week period was determined using Cox proportional hazards model. The model was adjusted to take into account two observations: 1) young adults (17-23 yrs) had an increased

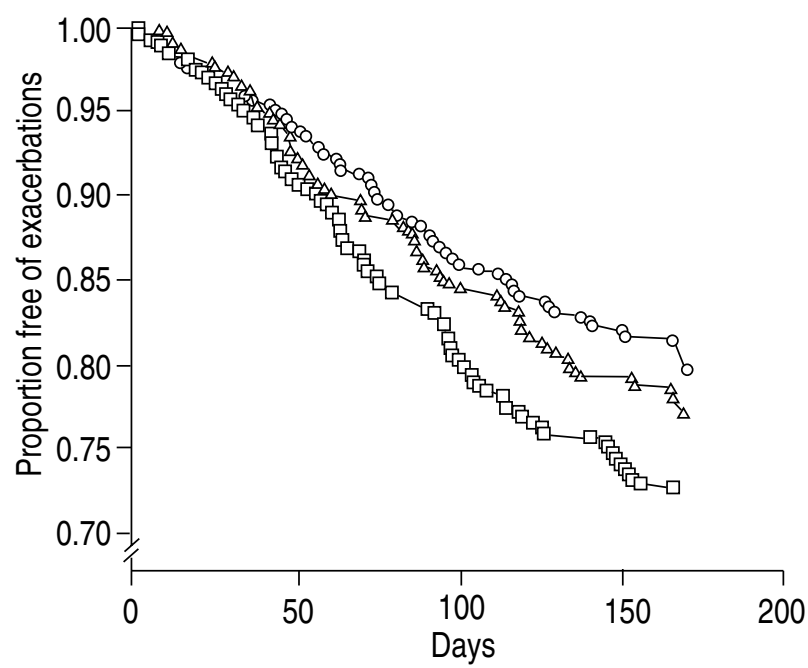

Fig. 3 - Cumulative proportion of patients who remained free of respiratory tract infectious exacerbations requiring parenteral antibiotic therapy by treatment group during Phase 3 double-blind trial. $-0-$ : rhDNase b.i.d.; $-\checkmark$ : rhDNase q.d.; $-\square-$ : placebo. rhDNase: recombinant deoxyribonuclease. (By kind permission of $N$ Engl J Med [22]). 
Table 3. - Age-adjusted relative risk of protocol-defined respiratory tract infections requiring parenteral antibiotics compared to placebo

\begin{tabular}{lcc}
\hline & \multicolumn{2}{c}{ rhDNase } \\
& Once daily & Twice daily \\
$\mathrm{n}=322$ & $\mathrm{n}=321$ \\
\hline Risk cp placebo & 0.72 & 0.63 \\
95\% confidence interval & $0.52-0.98$ & $0.46-0.87$ \\
p-value & 0.04 & $<0.01$ \\
\hline
\end{tabular}

cp: compared to; rhDNase: recombinant deoxyribonuclease [22].

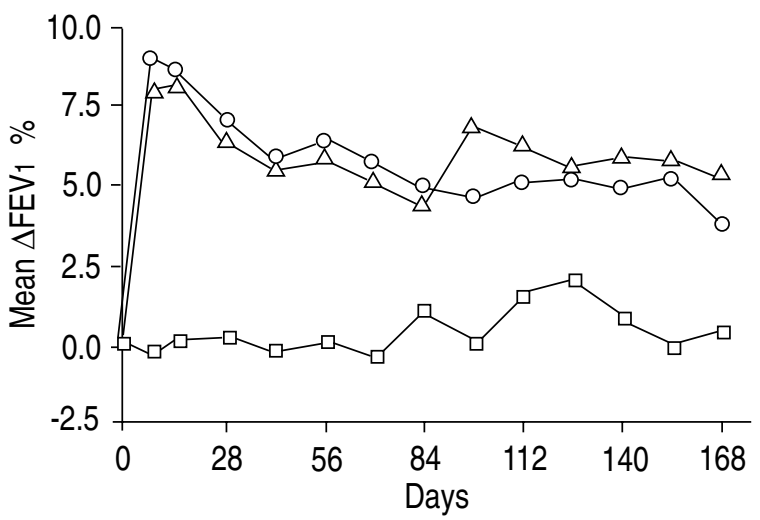

Fig. 4. - Mean percentage change from baseline in forced expiratory volume in one second $(\triangle \mathrm{FEV} 1)$ by treatment group during Phase 3 double-blind trial. $-{ }_{-}-$:rhDNase b.i.d.; $-\triangle-$ :rhDNase q.d; $-\square-$ : placebo. rhDNase: recombinant deoxyribonuclease. (By kind permission of $N$ Engl J Med [22]).

risk of pulmonary exacerbations; and 2) more patients in this age group had been randomized to receive rhDNase daily than had been allocated to the placebo group. Compared to placebo-treated patients, the age adjusted relative risk of protocol-defined respiratory tract infections requiring parenteral antibiotics is shown in table 3 . Thus, the risk of developing an exacerbation relative to placebo is reduced by $28 \%$ with rhDNase daily, and $37 \%$ with rhDNase b.i.d.

The trial showed that rhDNase significantly improved FEV1 from baseline compared with placebo (fig. 4), the mean percentage improvement was 5.8 and $5.6 \%$ for once daily and twice daily, respectively $(\mathrm{p}<0.001$ for each, compared with placebo). The value for FEV 1 in the placebo group was fairly stable throughout the trial. Patients whose FEV1 showed $>5 \%$ improvement over baseline after 2 weeks treatment with rhDNase were compared with patients who improved $<5 \%$ over baseline. The results indicated that even patients with minimal improvement in lung function had a reduction in exacerbations of respiratory tract infections. Compared with the placebo group, patients treated with rhDNase once daily spent 1.3 fewer days in hospital $(\mathrm{p}=0.06), 2.7$ fewer days receiving parenteral antibiotics $(\mathrm{p}<0.05)$, and 1.5 fewer days at home because of CF-related illness $(\mathrm{p}<0.05)$. Patients treated with rhDNase twice daily, spent 1.0 fewer days in hospital $(\mathrm{p}<0.05), 2.2$ fewer days receiving parenteral antibiotics $(\mathrm{p}<0.05)$, and 0.3 fewer days at home due to $\mathrm{CF}$-related illness $(\mathrm{p}=0.13)$. The rhDNase treated patients had less dyspnoea, improved well-being, and fewer CF-related symptoms.

\section{Safety results}

Administration of rhDNase did not cause allergy or anaphylaxis. There was no increased frequency of death, major respiratory complications of $\mathrm{CF}$ (e.g. dyspnoea, haemoptysis, pneumothorax), anaphylaxis or allergic reactions relative to placebo during the 24 week trial. By the end of the trial, antibiodies to rhDNase were detectable in $3 \%$ of patients treated daily, and $4 \%$ treated twice daily. This had no effect on clinical efficacy. There was no significant rise in concentrations of serum DNase 1 in the treatment groups as opposed to the placebo group, indicating the absence of significant accumulation of rhDNase during chronic exposure. The one type of adverse reaction, voice alteration, was increased in frequency when compared to placebo (7\% for placebo, $12 \%$ once daily, and $16 \%$ twice daily). This was rarely severe and was self-limiting. It was concluded that in patients with $\mathrm{CF}$ rhDNase improves airway function and reduces the risk of exacerbations of respiratory tract infection, and it was safe when administered for a period of 24 weeks.

\section{Open-label extension studies}

Eight hundred and eighty seven patients who had completed the double-blind study took part in the open extension. They received rhDNase $2.5 \mathrm{mg}$ b.i.d. in addition to their routine $\mathrm{CF}$ care. The results showed that the effect of rhDNase was maintained for at least 48 weeks of treatment (fig. 5). Regardless of the treatment group the patient had been assigned in the double-blind study, a smaller proportion of patients receiving rhDNase during the open-label extension required parenteral antibiotics for exacerbations of infection than the placebo-treated group during the double-blind trial. Groups receiving rhDNase during the double-blind study maintained the same improvement of FEV1 during the open extension. Patients who received placebo during the double-blind study showed an increase in FEV1 following initiation of treatment with rhDNase during the open-extension

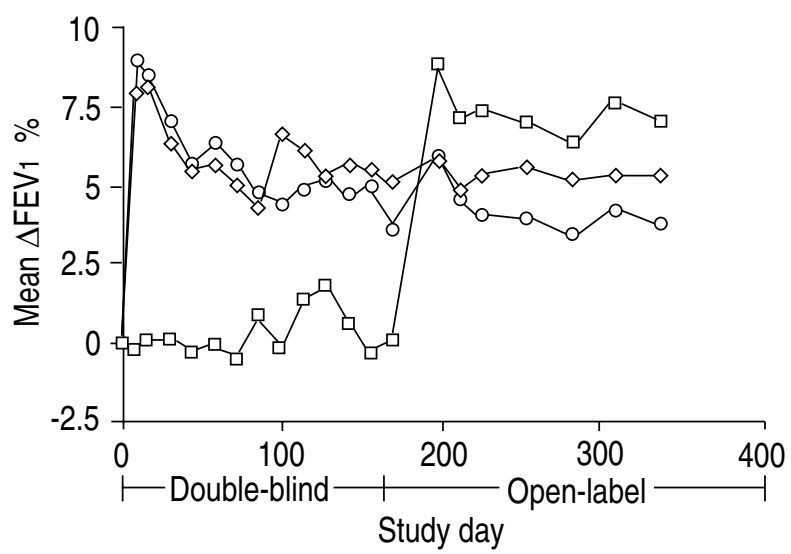

Fig. 5 - Mean percentage in forced expiratory volume in one second $\left(\triangle F E V_{1}\right)$ from baseline in the Phase 3 trial and open-label period. $-\square-$ : placebo/Dornase alfa $2.5 \mathrm{mg}$ b.i.d.; $\checkmark$ : Dornase alfa 2.5 $\mathrm{mg}$ q.d./2.5 mg b.i.d.; ${ }^{-}-$: Dornase alfa $2.5 \mathrm{mg}$ b.i.d. $/ 2.5 \mathrm{mg}$ b.i.d. 
study. Safety assessments during this 24 week openlabel treatment showed that the incidence of adverse events was similar to that reported during the doubleblind study.

The authors have followed the patients who participated in the UK Phase 2 study who have continued to receive open-label rhDNase for 24 months. The mean improvement in FEV1 is $7.1 \%$ and FVC $4.2 \%$. There was also some improvement in body weight in these adult patients [23].

\section{Trials involving severely ill patients}

Patients with severe pulmonary disease comprise about $7 \%$ of the CF population. These patients have a FVC of $<40 \%$ pred [3]. The studies so far reported involved patients with mild or moderate pulmonary disease, FVC $\geq 40 \%$ pred. To study the effect of rhDNase on severely ill patients, a multicentre study was carried out in which 70 patients were randomized to receive placebo or rhDNase $2.5 \mathrm{mg}$ b.i.d. for 14 days. All patients had a FVC of $<40 \%$ pred. During the study, the lung function in both groups improved, but there was no significant difference between the placebo and the DNase treated group. There was, however, no increase in intercurrent events in the group treated with rhDNase. The patients who completed this study were then eligible to enter a 24 week open-label extension study. All patients were treated with rhDNase $2.5 \mathrm{mg}$ b.i.d. Thirty nine patients completed the extension study. When all patients who entered the study are considered, there was a mean improvement in FEV1 and FVC of 9.3 and $17.8 \%$, respectively. It would appear that these more severely affected patients may take longer to benefit from treatment with rhDNase [24]. A further double-blind study of 3 months duration comparing placebo and rhDNase is in progress on severely ill patients [25].

\section{Nebulizer studies}

Three nebulizer systems were studied to see if they produced the same clinical results. They were the Hudson T Updraft II, and the Marquest Acorn II used in conjunction with a Pulmo-Aide compressor, and the reusable Pari LC used in conjunction with the Proneb compressor. Nebulizers and compressors were used in accordance with the manufacturers' instructions. The results are shown in table 4 [26]. The three nebulizer systems gave comparable results.

In conclusion, trials have shown the efficacy and safety of rhDNase at a dose of $2.5 \mathrm{mg}$ daily or twice daily among clinically stable patients with $\mathrm{CF}$, who were $>5$ yrs of age and had a baseline FVC of $>40 \%$ predicted value. Inhalation of rhDNase has been shown to reduce the incidence of exacerbations of respiratory tract infections and to improve pulmonary function over a 24 week double-blind study and an additional 24 week open-label treatment. Recombinant DNase was well-tolerated by cystic fibrosis patients and was not associated with an
Table 4. - Nebulizer comparison trial

\begin{tabular}{lccc}
\hline & \multicolumn{3}{c}{$\%$ change from baseline FEV1 } \\
\cline { 2 - 4 } Nebulizer & Day 8 & Day 15 & Mean \\
\hline T Updraft II $(\mathrm{n}=132)$ & $13.8 \pm 1.4$ & $13.0 \pm 1.5$ & $13.2 \pm 1.4$ \\
Acorn II $(\mathrm{n}=134)$ & $14.3 \pm 1.4$ & $14.1 \pm 1.5$ & $14.1 \pm 1.5$ \\
LC $(\mathrm{n}=131)$ & $13.6 \pm 1.5$ & $12.5 \pm 1.5$ & $13.2 \pm 1.4$ \\
\hline
\end{tabular}

FEV1: forced expiratory volume in one second.

increased incidence of serious respiratory or other adverse events. There was no evidence of serious drug allergy or anaphylaxis. A small proportion of patients developed serum antibodies, but in none has this been associated with allergy or anaphylaxis, even upon rechallenging. There was a small numerical increase in the incidence of voice alteration, pharyngitis and largyngitis in patients receiving rhDNase as compared with placebo but this was usually mild and transient. It is recommended that DNase is given in a dose of $2.5 \mathrm{mg}$ daily by jet nebulizer. Nebulizer systems which have been fully assessed are the Hudson T Updraft II, or the Marquest Acorn II used in conjunction with Pulmo-Aide compressor, and the reuseable Pari LC used in conjunction with the Proneb compressor. Short-term trials have not shown clinical benefit in patients whose FVC is $<40 \%$. However, the safety of DNase has been shown in these $7 \%$ of sicker patients. The clinician may, therefore, wish to treat patients with an FVC of $<40 \%$ predicted, or await the result of the ongoing double-blind study in this group of patients. There are currently no data available on patients less than 5 yrs of age.

The extensive clinical trial evidence reviewed above shows that rhDNase, although not a cure, is an important addition to the routine treatments available for patients with CF who have evidence of inflammatory lung disease.

\section{References}

1. Penketh ARL, Wise A, Mearns MB, Hodson ME, Batten JC. Cystic fibrosis in adolescents and adults. Thorax 1987; 42: 526-532.

2. Andersen DH. Cystic fibrosis of the pancreas and its relation to celiac disease: a clinical and pathological study. Am J Dis Child 1938; 56: 344-399.

3. FitzSimmons S. The changing epidemiology of cystic fibrosis. J Pediatra 1993; 122: 1-9.

4. Dodge JA, Morison S, Lewis PA, et al. Cystic fibrosis in the United Kingdom 1968-1988: incidence, population and survival. Paediatr Perinat Epidemiol 1993; 7: 157-166.

5. Chernick WS, Barbero GJ. Composition of tracheobronchial secretions in cystic fibrosis of the pancreas and bronchiectasis. Pediatrics 1959; 24: 739-745.

6. Potter J, Matthews LW, Lemm J, Spector JS. Composition of pulmonary secretions from patients with and without cystic fibrosis. Am J Dis Child 1960; 199: 493-495.

7. Matthews LW, Spector S, Lemm J, Potter JL. Studies on pulmonary secretions. I. The overall composition of secretions from patients with cystic fibrosis, bronchiectasis and laryngectomy. Am Rev Respir Dis 1963; 88: 199-204. 
8. Picot R, Das I, Reid L. Pus deoxyribonucleic acid and sputum viscosity. Thorax 1978; 33: 235-242.

9. Armstrong JB, White JC. Liquification of viscous purulent exudates by deoxyribonuclease. Lancet 1950; 2 : 739-742.

10. Chernick WS, Barbero GJ, Eichel JH. In vitro evaluation of effect of enzymes on tracheobronchial secretions from patients with cystic fibrosis. Pediatrics 1961; 27: 589-596.

11. Elmes PC, White JC. Deoxyribonuclease in the treatment of purulent bronchitis. Thorax 1953; 8: 295-300.

12. Salomon A, Herschfils JA, Segal MD. Aerosols of pancreatic dornase in bronchopulmonary disease. Ann Allergy 1954; 12: 71-79.

13. Spier R, Witebsky E, Paine JR. Aerosolised pancreatic dornase and antibiotics in pulmonary infections. $J A m$ Med Assoc 1961; 178: 878-886.

14. Lieberman J. Dornase aerosol effect on sputum viscosity in cases of cystic fibrosis. J Am Med Assoc 1968; 205: 312-313.

15. Raskin P. Bronchospasm after inhalation of pancreatic dornase. Am Rev Respir Dis 1968; 98: 697-698.

16. Shak S, Capon DJ, Hellmiss R, Marsters SA, Baker CL. Recombinant human DNase 1 reduces the viscosity of cystic fibrosis sputum. Proc Natl Acad Sci USA 1990; 87: 9188-9192.

17. Rubin BK, Ramirez OE, Baharav AL. The physical and transport properties of CF sputum after treatment with rhDNase. Ped Pulmonol 1993; 16 (Suppl. 9): 251 (Abstract).

18. Aitken ML, Burke W, McDonald G, et al. Recombinant human DNase inhalation in normal subjects and patients with cystic fibrosis. Phase 1 study. J Am Med Assoc 1992; 267: 1947-1951.
19. Hubbard RC, McElvaney NF, Birrer P, et al. A preliminary study of aerosolised recombinant human deoxyribose 1 in the sputum of cystic fibrosis. $N$ Engl J Med 1992; 326: 812-815.

20. Ramsey BW, Astley SJ, Aitken ML et al. Efficacy and safety of short-term administration of aerosolised recombinant human deoxyribonuclease in patients with cystic fibrosis. Am Rev Respir Dis 1993; 148: 145151.

21. Ranasinha C, Assoufi B, Shak S et al. Efficacy and safety of short-term administration of aerosolised recombinant human DNase 1 in adults with stable stage cystic fibrosis. Lancet 1993; 342: 199-202.

22. Fuchs HJ, Borowitz DS, Christiansen PH et al. Efficacy of aerosolised recombinant human DNase on exacerbations of respiratory symptoms and on pulmonary function in patients with cystic fibrosis. N Engl J Med 1994; 331: 637-642.

23. Shah PL, Scott SF, Geddes DM, Hodson ME. Two years experience with recombinant human DNase 1 in the treatment of pulmonary disease in cystic fibrosis. Respir Med 1995; 89: 499-502.

24. Shah PL, Bush A, Canny GJ et al. Recombinant human DNase 1 (rhDNase) in cystic fibrosis patients with severe pulmonary disease: a short-term, double-blind study followed by a six month open-label treatment. Eur Respir $J$ 1995; 8: 954-958.

25. Johnson C. A multicentre, double blind, placebo-controlled study to evaluate the safety and efficacy of aerosolised rhDNase therapy in patients with cystic fibrosis who have advanced lung disease. Pediatr Pulmonol 1994; (Suppl 10): 115.

26. Data on file. Genentech Inc. South San Francisco, CA, USA. 